\title{
A large-scale genomic approach affords unprecedented resolution for the molecular epidemiology and evolutionary history of contagious caprine pleuropneumonia
}

\author{
Virginie Dupuy $^{1,2^{*}}$ (D) Axel Verdier ${ }^{1,2}$, François Thiaucourt ${ }^{1,2}$ and Lucía Manso-Silván ${ }^{1,2}$
}

\begin{abstract}
Contagious caprine pleuropneumonia (CCPP), caused by Mycoplasma capricolum subsp. capripneumoniae (Mccp), is a devastating disease of domestic goats and of some wild ungulate species. The disease is currently spreading in Africa and Asia and poses a serious threat to disease-free areas. A comprehensive view of the evolutionary history and dynamics of Mccp is essential to understand the epidemiology of CCPP. Yet, analysing the diversity of genetically monomorphic pathogens, such as Mccp, is complicated due to their low variability. In this study, the molecular epidemiology and evolution of CCPP was investigated using a large-scale genomic approach based on next-generation sequencing technologies, applied to a sample of strains representing the global distribution of this disease. A highly discriminatory multigene typing system was developed, allowing the differentiation of 24 haplotypes among 25 Mccp strains distributed in six genotyping groups, which showed some correlation with geographic origin. A Bayesian approach was used to infer the first robust phylogeny of the species and to date the principal events of its evolutionary history. The emergence of Mccp was estimated only at about 270 years ago, which explains the low genetic diversity of this species despite its high mutation rate, evaluated at $1.3 \times 10^{-6}$ substitutions per site per year. Finally, plausible scenarios were proposed to elucidate the evolution and dynamics of CCPP in Asia and Africa, though limited by the paucity of Mccp strains, particularly in Asia. This study shows how combining large-scale genomic data with spatial and temporal data makes it possible to obtain a comprehensive view of the epidemiology of CCPP, a precondition for the development of improved disease surveillance and control measures.
\end{abstract}

\section{Introduction}

Contagious caprine pleuropneumonia (CCPP) is a severe respiratory disease affecting goats and some wild ruminant species. The disease, listed by the World Organisation for Animal Health (OIE) [1] has a great economic impact on livestock production in fragile rural economies and poses a serious threat to disease-free areas. CCPP is caused by Mycoplasma capricolum subsp. capripneumoniae (Mccp), a member of the Mycoplasma mycoides cluster [2]. This cluster comprises five mycoplasmas which are pathogenic for ruminants, including Mycoplasma capricolum subsp. capricolum (Mcc), the closest relative of Mccp, and Mycoplasma mycoides

\footnotetext{
* Correspondence: virginie.dupuy@cirad.fr

'CIRAD, UMR CMAEE, F-34398 Montpellier, France

2INRA, UMR1309 CMAEE, F-34398 Montpellier, France
}

subsp. mycoides (Mmm), the agent of contagious bovine pleuropneumonia (CBPP). Since it was first isolated in 1976 [3], Mccp has only been isolated in 17 countries, mainly because of its fastidiousness in culture. However, clinical descriptions have been published in nearly 40 countries in Africa and Asia, suggesting a much wider distribution [4]. The disease is present in the Arabian Peninsula, North, Central and East Africa and Asia, but its boundaries are still uncertain, particularly in western and southern Africa and in Asia. In the last decade, an increasing number of outbreaks have been reported in both domestic and wild ruminants [5]. New detections were often the result of improved diagnosis, confirming the presence of the disease in suspected regions [6-8], but in certain cases, they indicated that the disease had spread to new territories $[9,10]$. 
A comprehensive view of the evolutionary history and the dynamics of Mccp strains is essential to understand the epidemiology of CCPP. Molecular epidemiology investigations require robust genotyping tools with sufficient resolution, while evolutionary analyses are also constrained by the need for a reliable molecular clock to infer evolutionary timescales. Various molecular methods have been developed for the analysis of Mccp strains. The first study on the molecular evolution of Mccp was based on the $16 \mathrm{~S}$ rRNA gene [11], which provided a basis for the phylogeny and systematics of bacteria since the evolutionary studies of Woese [12]. Mccp strains showed a surprisingly high degree of polymorphism between their two 16S rRNA gene copies [13], which allowed the use of this housekeeping gene to combine epidemiological and evolutionary analyses. Still, the study was limited by the low discriminatory power of this molecular marker. Despite the heterogeneity observed in the sequence of its two rRNA operons, the Mccp genome has been shown to be rather monomorphic. Thus, a phylogenic study of the M. mycoides cluster based on five partial housekeeping gene sequences showed a very low distance between Mccp strains [14]. The low diversity of such a monomorphic bacterium precluded the use of standard multilocus sequence typing (MLST), which relies on housekeeping genes [15]. Approaches based on alternative sequences, independently of their coding capacity, were thus preferred. Analysis of the H2 locus allowed the discrimination of four groups showing a good correlation with geographic origin [16]. This system was improved by the addition of seven loci, increasing the discriminatory power and leading to the description of five groups [4]. Although this multilocus sequence analysis (MLSA) scheme targets polymorphic loci by focusing on non-housekeeping genes (e.g., intergene regions, pseudogenes) it interrogates less than $1 \%$ of the genome and therefore has limited discriminatory power for epidemiological investigations. Moreover, it is not reliable for phylogenetic analyses because of differences in the molecular evolutionary clock among target sequences.

These limitations can now be overcome by the recent development of high-throughput methods [17]. Even though single nucleotide polymorphism (SNP) frequencies are low in monomorphic pathogens, their numbers can be dramatically increased by enlarging the scale of the analysis. Using high-throughput data can enhance genetic investigations by providing a way to disclose genome-wide variations. Combining large-scale genomic data with spatial and temporal data already enabled a comprehensive view of the molecular epidemiology and evolution of bacterial pathogens like Salmonella typhi [18], Yersinia pestis [19], Mmm [20] and Mycobacterium tuberculosis [21]. Following the increasing use and affordability of DNA sequencing with next-generation sequencing (NGS) technologies, complete annotated genomes of several Mccp strains have become available [22-24], thereby making large-scale genomic investigations possible.

In this study, we investigated the molecular epidemiology and evolution of CCPP using a large-scale genomic approach based on NGS data, on a sample of strains representing the global distribution of this disease. Our main objective was to develop a discriminatory genotyping method to investigate the genetic diversity and population structure of Mccp. A robust phylogeny was also inferred from a large phylogenomic data set and divergence time of Mccp strains was estimated to reconstruct the evolutionary history of CCPP.

\section{Materials and methods Sampling}

The 25 strains analysed in this study are summarised in Table 1. This collection includes 15 strains isolated in Central (4)/East (11) Africa, four strains from the Arabian Peninsula, four strains from the Mediterranean Basin and two strains from Central/East Asia in attempts to encompass the known global diversity of this pathogen. They are all epidemiologically unrelated isolates and most of them were previously analysed by traditional genotyping systems $[4,11,16]$.

\section{Sample preparation and sequencing}

Twenty-one strains for which the genome sequence was not available were cultured in modified Hayflick's medium [14] at $37{ }^{\circ} \mathrm{C}, 5 \% \mathrm{CO}_{2}$. Culture purity was ensured by phenotypic control on solid medium and specific Mccp QPCR amplification [25]. DNA was extracted using a standard phenol/chloroform method [26]. DNA purity, quality and quantity were checked using NanoDrop $^{\text {Tw }}$ ND-1000 Spectrophotometer (Thermo Fisher Scientific, MA, USA), gel electrophoresis and Qubit $^{\circ} 2.0$ fluorometer (Invitrogen, USA), respectively. Then, 21 tagged standard genomic libraries were constructed and pooled to be sequenced in $100 \mathrm{bp}$ single reads on an Illumina HiSeq 2000 (GATC, Constanz, Germany).

\section{Gene selection}

The choice of Mccp genes was based on the choice previously made for the analysis of the evolutionary history of Mmm [20], consisting in 62 rigorously selected genes. Pseudogenes and duplicated genes had been excluded from this set, as well as genes coding for membrane proteins or restriction enzymes, and those known to be involved in horizontal transfer. The annotated, circularised genome sequence of strain 9231-Abomsa [23] (Table 1) was used as reference. Four genes did not exist in the Mccp genome (guaC, gntR, suk, bgl), while dnaC was duplicated and was therefore excluded. As a result, a 
Table 1 List of Mycoplasma capricolum subsp. capripneumoniae strains analysed and corresponding MLSA types

\begin{tabular}{|c|c|c|c|c|c|c|c|}
\hline Strain & Year & Country & Location & World Region & Supplier & Accession number & MLSA type \\
\hline 97095-Tigray & 1995 & Ethiopia & Tigray & East Africa & NVI-E & & $1-010$ \\
\hline 99108-P1 & 1999 & Ethiopia $^{a}$ & Tigray & East Africa & - & GenbaNK:JMJI00000000 & $1-010$ \\
\hline 04012 & 2004 & Qatar & Doha, Al Wabra & Arabian Peninsula & AWWP & & $1-010$ \\
\hline M79/93 & 1993 & Uganda & East & East Africa & NVI-S & & $1-020$ \\
\hline |LRI181 & 2012 & Kenya & NK & East Africa & - & GenbaNK:LN515399 & $1-030$ \\
\hline 8789 & 1987 & Chad & Karal, Dandi & Central Africa & LRVZF & & $2-010$ \\
\hline 94156 & 1994 & Chad & N'Djamena & Central Africa & LRVZF & & $2-010$ \\
\hline 05021 & 2005 & Sudan & Darfur, Nyala & Central Africa & VRA & & $2-010$ \\
\hline 95043 & 1995 & Niger & Goure & Central Africa & LABOCEL & & $2-020$ \\
\hline $44 \mathrm{~F} 04$ & 2004 & Turkey & Thrace & Mediterranean Basin & VLA & & $3-020$ \\
\hline 12002 & 2012 & Tajikistan & Farkhor & Central Asia & CIRAD & & $3-020$ \\
\hline C550/1 & 1991 & UAE & Dubai & Arabian Peninsula & CVRL & & $3-030$ \\
\hline M1601 & 2007 & China & Gansu & East Asia & - & GenbaNK:CM001150 & $3-010$ \\
\hline Gabes & 1980 & Tunisia & Gabes & Mediterranean Basin & CIRAD & & 4-010 \\
\hline $9081-487 P$ & 1990 & Oman & NK & Arabian Peninsula & MAF-O & & 4-010 \\
\hline 07033 & 2007 & Turkey & Elazig & Mediterranean Basin & FU & & 4-010 \\
\hline $7 / 2$ & 1986 & Turkey $^{a}$ & NK & Mediterranean Basin & MRI & & $4-020$ \\
\hline 97097-Errer & 1997 & Ethiopia & Errer & East Africa & NVI-E & & $5-010$ \\
\hline Yatta B & 1997 & Kenya & Yatta & East Africa & NVI-S & & $5-020$ \\
\hline AMRC-C758 & 1981 & Sudan & NK & East Africa & $\mathrm{AU}$ & & $5-020$ \\
\hline F38 & 1976 & Kenya & NK & East Africa & Type strain & & $5-030$ \\
\hline $94029-C 5$ & 1994 & Oman & NK & Arabian Peninsula & AVS & & $5-040$ \\
\hline 91039-С3 & 1991 & Ethiopia & NK & East Africa & NVI-E & & $5-050$ \\
\hline 9231-Abomsa & 1982 & Ethiopia & Godjam & East Africa & - & GenbaNK:LM995445 & $5-060$ \\
\hline 92138-CLP1 & 1992 & Ethiopia & NK & East Africa & NVI-E & & 5-060 \\
\hline
\end{tabular}

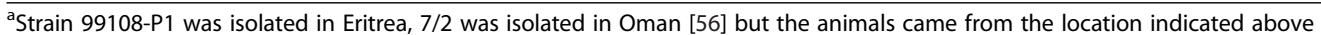

Abbreviations: AU Aarhus University, Denmark, AVS Agriculture and Veterinary Services, Oman, AWWP AI Wabra Wildlife Preservation, Qatar, CIRAD, France, CVRL Central Veterinary Research Laboratory, United Arab Emirates, FU Firat University, Turkey, LABOCEL Laboratoire Central de I'Elevage de Niamey, Niger, LRVZF Laboratoire de Recherches Vétérinaires et Zootechniques de Farcha, Chad, MAF-O Ministry of Agriculture and Fisheries, Oman, MRI Moredun Research Institute, UK, NVI-E National Veterinary Institute, Ethiopia, NVI-S National Veterinary Institute, Sweden, VRA Veterinary Research Administration, Sudan, NK not known

subset of 57 genes, comprising 47 coding sequences and 10 pseudogenes of the core genome, were used (Additional file 1). The 57 genes are evenly distributed along the chromosome of strain 9231-Abomsa (Additional file 2).

\section{Data set collection}

The sequences of the 57 selected genes from strain 9231Abomsa, including flanking regions (up to $350 \mathrm{bp}$ ), were concatenated and annotated, resulting in an "enlarged" sequence of $107050 \mathrm{bp}$ (Additional file 3). This sequence was used as reference to automatically retrieve the entire gene set from the whole genome sequence data of 21 Mccp strains by mapping raw data using Seqman NGen (2.0) software (DNASTAR, Madison WI, USA). First, this procedure allowed the correct mapping of reads on the entire sequence of each corresponding gene, thanks to the presence of flanking regions. Second, it allowed the visual verification of sequencing depth and the identification of any incongruities on all coding sequences in the Seqman genome browser. On average, a read depth of 500X was obtained. SNPs and indels were called when more than $85 \%$ of the reads supported the change.

An in-house software was developed at CIRAD Montpellier to extract and concatenate the gene sequences corresponding to each strain from the enlarged reference sequence. Sequence searches are based on the NeedlemanWunsch algorithm, using tags to frame sequences of interest. These tags are short sequences homologous to the extremities of each target gene. The algorithm described here is implemented in $\mathrm{C}++$ as a stand-alone program, "SelectRegion", and the source is freely available from the authors on request. The process can also be automated using graphical-interfaces within the web-based Galaxy [27]. 
Otherwise, sequence data were retrieved from the published genome sequences of strains M1601, 99108 and ILRI181 (Table 1).

\section{Genotyping analyses}

For diversity analyses, the 57 genes (comprising both coding sequences and pseudogenes) corresponding to each of the 25 strains analysed were selected and concatenated as described above. Sequences were aligned using Clustal W with default parameters (Additional file 4). Haplotypes were estimated taking into account all sites, including gaps, removing invariant sites, with DnaSP [28]. A median-joining network was reconstructed using NETWORK V4.6 [29].

The discriminatory power of the genotyping system was calculated using Simpson's index of diversity [30], which expresses the probability of two unrelated strains sampled from the test population being placed into different genotyping groups. A total of $95 \%$ confidence intervals (CI) were determined as previously described [31].

\section{Phylogeny and molecular dating analyses}

From the 57 genes initially selected, a subset of 47 coding sequences was retained for evolutionary analyses. The 47 genes of 25 Mccp strains were extracted from each corresponding enlarged reference sequence using the internal software SelectRegion. The type strain of the subspecies Mcc (California $\operatorname{Kid}^{\mathrm{T}}$ ), which is the closest relative of Mccp, was chosen as outgroup and corresponding sequence data were obtained from the published genome sequence [Genbank:CP000123].

Sequences were aligned using Clustal W with default parameters (Additional file 5). The Tamura-Nei, 93 (TN93) model was selected as the best fitting model by Modeltest V3.7 [32]. A maximum-likelihood phylogenetic tree was inferred using PhyML V3.0 [33] on the Galaxy web platform. A bootstrap resampling procedure with 1000 replicates was used to assess the reliability of key tree nodes. To infer a temporal framework from dated sequences, a Bayesian approach, implemented in the flexible Bayesian phylogenetic analysis package BEAST V1.6.2 [34], was used. This allowed the simultaneous estimation of the tree structure, the time of the most recent common ancestor (MRCA), the divergence time of nodes, and the mutation rate. The TN93 evolution model and partition codon $(1+2,3)$ were selected. Two molecular clocks (strict-clock and uncorrelated lognormal-clock) and various demographic models (constant, expansion, exponential and extended Bayesian skyline plot) were tested. Convergence was evaluated using Tracer V1.6. To choose the best fitting clock and demographic model, the Bayes Factors [35,36] were calculated from the marginal likelihoods on the Akaike's information criterion through MCMC (AICM) using
Tracer V1.6. The Maximum Clade Credibility tree was constructed using TreeAnnotator V1.6 and visualised using FigTree V1.3.

\section{Results}

Genetic diversity and molecular genotyping of Mccp strains A discriminatory genotyping system based on a largescale genomic approach was developed to characterise the diversity of Mccp by analysing 25 strains representing the known global distribution of this species (Table 1). A set of 57 genes, comprising 47 coding sequences and 10 pseudogenes (Additional file 1), was analysed, covering 77898 base pairs $(7.7 \%$ of the genome). Fifty-two of these genes were polymorphic as a result of 239 polymorphic positions consisting in 212 SNPs and 17 indels (Additional file 6). Nine events corresponded to indels of either one/two bases or one/two codons and eight events to variations in homopolymer size. The average frequency of polymorphic sites in the gene set was of one event per $577 \mathrm{pb}$ for coding sequences, and one event per $210 \mathrm{pb}$ for pseudogenes. The 239 polymorphic positions made it possible to define 24 haplotypes among the 25 strains analysed, resulting in a Simpson's diversity index of 0.997 (0.988-1.000). Polymorphic positions consisted in 115 informative sites and 124 sites specific to single haplotypes.

A single network connecting all strains was drawn using NETWORK (Figure 1). The 24 haplotypes were structured in six genotyping groups, named group A to F. Group A was quite homogeneous and included four extremely similar strains from East Africa and a more distant strain from the Arabian Peninsula. Within this group, M79/93 and 99108-P1 were the only strains that could not be distinguished. Four strains from Central Africa, also showing little diversity, constituted group B. Group $C$ included three rather variable strains from the Mediterranean Basin, the Arabian Peninsula and Central Asia. Chinese strain M1601, positioned at the extremity of a long isolated branch, was designated group D, and was the only representative of this group. Group E showed little variability and comprised three strains originating from the Mediterranean Basin and a strain from the Arabian Peninsula. Finally, Group F, which was the most populated and variable group, included seven strains from East Africa and one strain from the Arabian Peninsula.

Three groups (A, B and F) presented at least four isolates originating from the same geographic region, whereas too few strains from groups D and $\mathrm{E}$ were available to confirm a correlation between genotyping group and geographic origin. In group $\mathrm{C}$ no clear correlation could be found with geographic origin, also arguably due to insufficient sampling. In the Arabian Peninsula many different groups (all except B and D) were found and in 


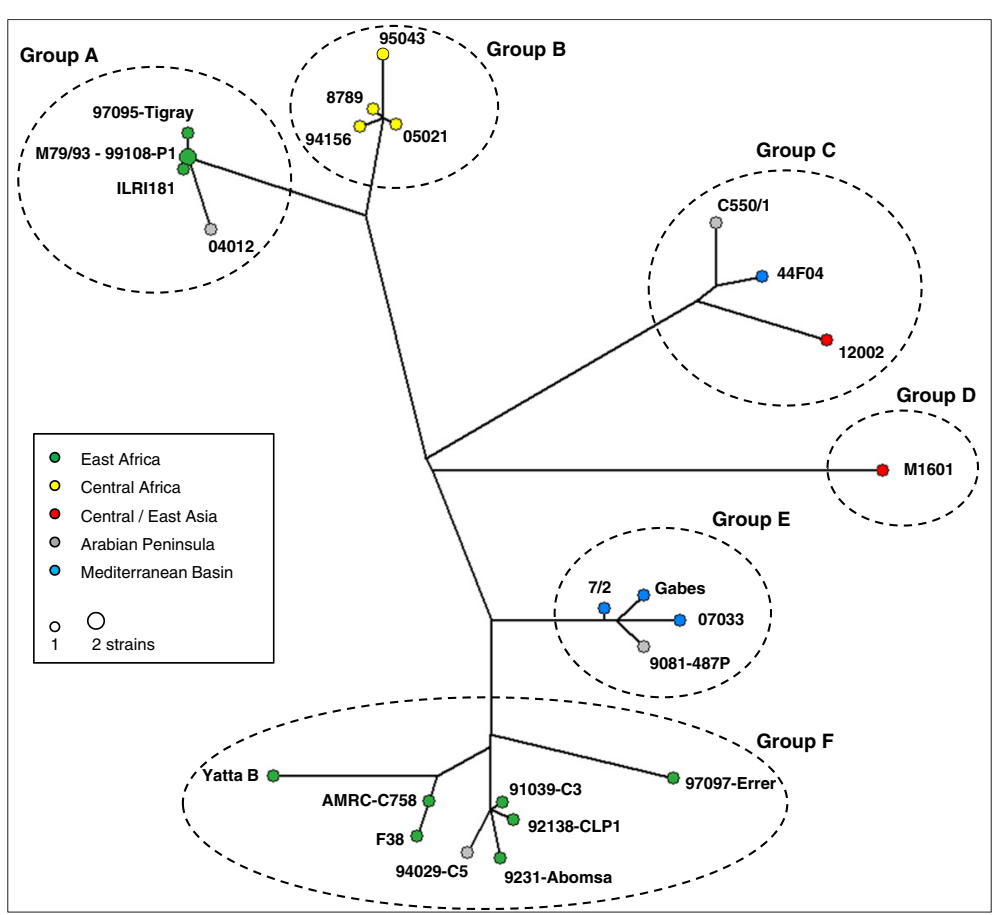

Figure 1 Haplotype network of Mycoplasma capricolum subsp. capripneumoniae. The median-joining network was reconstructed using NETWORK based on the alignment of 57 concatenated genes from 25 Mccp strains. The tree shows 24 haplotypes (nodes) resulting from 239 polymorphic positions including gaps. Different colours indicate the geographic origin of haplotypes.

Turkey two groups (C and E) were present. Also, two distinct groups (A and F) were found in East Africa.

\section{Evolutionary history of Mccp}

A robust phylogeny of Mccp was reconstructed based on high-throughput genomic data of 25 selected Mccp strains (Table 1) and an Mcc outgroup. Among the 57 genes previously analysed for genotyping, a set of 47 coding sequences was retained, while pseudogenes were excluded (Additional file 1) to minimise molecular clock variation and homoplasy. After alignment of the sequences, robust trees were inferred based on 134 SNPs using both maximum likelihood (Additional file 7) and Bayesian (Figure 2) approaches. Tree topologies were identical for supported branches. The six genotyping groups described above (group A to group F) were retrieved as clusters supported by high node support values. They formed two major lineages. Lineage I included group A, present in East Africa, and group B, present in Central Africa. Lineage II comprised groups C, D, E and F and was divided into two sub-lineages. The first sub-lineage comprised groups $\mathrm{C}$ and $\mathrm{D}$, representing Asian strains, while group E (representing strains from the Mediterranean Basin) and group F (comprising mainly strains from East Africa) were clustered in a second, heterogeneous sub-lineage. Divergence time for Mccp strains was estimated from the dated sequences using the flexible Bayesian phylogenetic analysis package BEAST (Figure 2). Bayes Factor tests indicated that the strict-clock model fitted the data better than the relaxed-clock model. In contrast, there was no substantial difference between population models. To exempt analyses from dependence on a pre-specified demographic model, the extended Bayesian skyline plot model was applied. The mean substitution rate for Mccp, estimated on 47 coding sequences, was $1.3 \times 10^{-6}$ (substitutions per site per year). The MRCA of Mccp strains emerged 269 years ago (95\% highest posterior densities (HPDs) intervals between 120 years and 736 years). Lineage II diverged around 230 years ago (95\% HPDs: 104-633) and dissociated into two sub-lineages dated $202(95 \%$ HPDs: 88-554) and 136 years ago (95\% HPDs: 67-360) respectively, while lineage I diverged around 159 years ago (95\% HPDs: 66-438). All genotyping groups except $\mathrm{D}$ were formed recently at almost the same period (between 19 and 222 years ago), with the most probable age being between 35 years (for group A) and 90 years (for group F). Indeed, group D is represented by a single strain (the only Chinese strain available so far) and, as long as the diversity of this group remains unresolved, it will not be possible to date its emergence. 


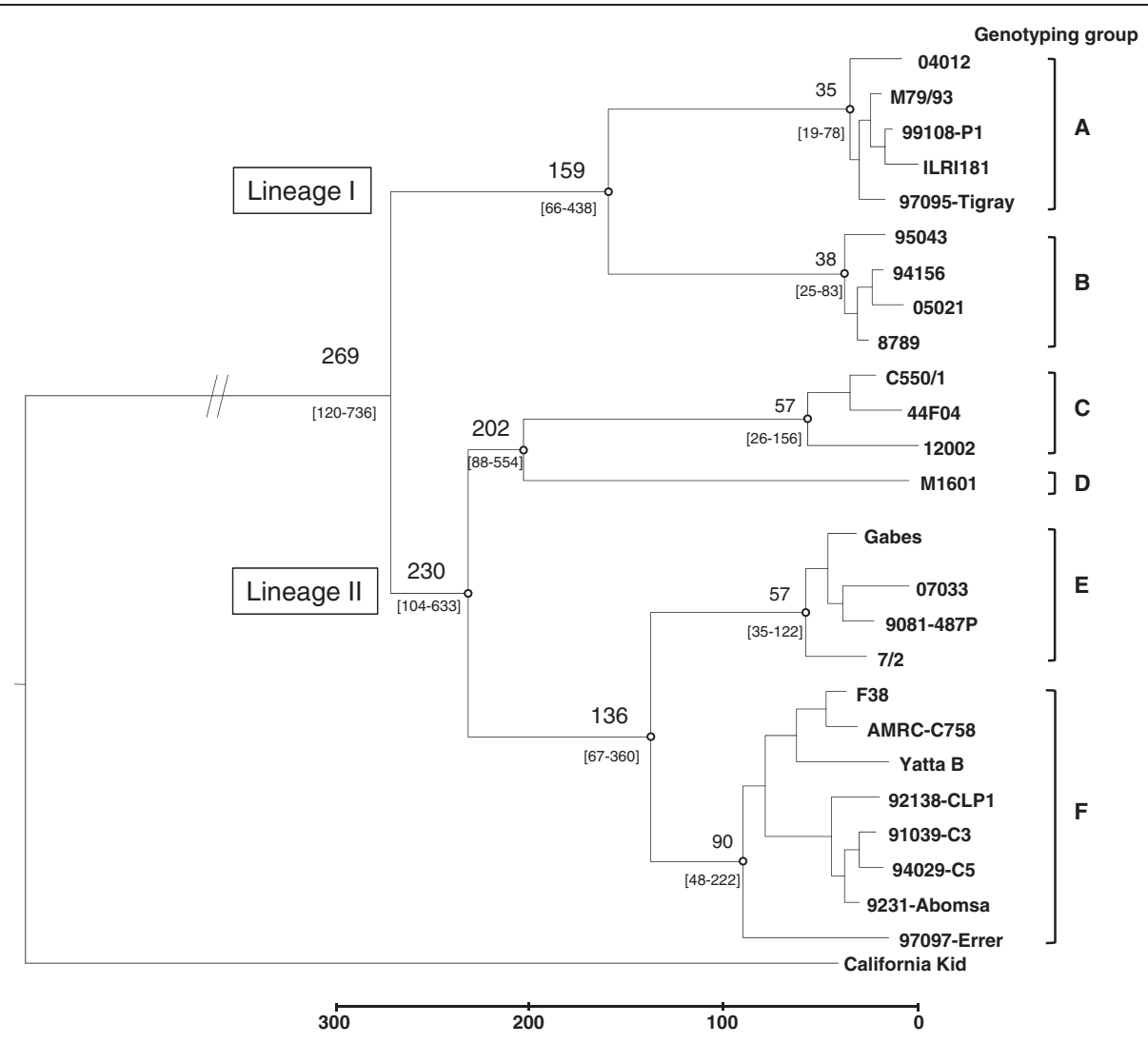

Figure 2 Bayesian inference of Mycoplasma capricolum subsp. capripneumoniae evolutionary history. The Maximum Clade Credibility tree resulted from BEAST analysis of the alignment of concatenated sequences of 47 coding sequences from 25 Mccp strains. Bayesian posterior probabilities higher than 0.9 are represented by a white circle. Age estimates are displayed for relevant nodes with the $95 \%$ highest probability density intervals in brackets. The scale is given in years before present. The branch corresponding to the outgroup (California Kid) was shortened, as indicated by parallel bars.

\section{Discussion}

Increasing the scale of analysis to tackle the genetic population structure and evolution of the monomorphic pathogen Mccp

Analysing the diversity in genetically monomorphic pathogens such as Mccp is a challenge because of their low variability. Classical molecular approaches based on sequencing a few gene fragments provide so little appreciable diversity that, to date, few questions regarding the epidemiology and evolution of CCPP have been answered. Technological advances in DNA sequencing now allow the rapid sequencing of complete bacterial genomes and offer the possibility to multiplex many different isolates, thereby dramatically increasing the discovery of polymorphic positions to analyse species diversity [37]. In this study, using data from NGS technologies made it possible to develop new approaches based on the analysis of a large number of genes.

The choice of genes for Mccp was based on a recent study of the evolutionary history of Mmm [20]. This pathogen, responsible for CBPP, is also a very monomorphic species for which classical methods like MLST do not afford sufficient discriminatory power. In this previous study, the rigorous selection of 62 genes from the core genome allowed the differentiation of 19 genotypes out of 20 strains analysed and made it possible to elucidate the evolutionary history of CBPP. Therefore, the same approach was chosen to investigate the molecular epidemiology and evolution of CCPP. In the present study, the initial set of 62 genes was reduced to 57 genes after exclusion of missing and duplicated genes in Mccp. The selection finally comprised 47 coding sequences and 10 pseudogenes of the core genome. However, it should be noted that the complete set of 57 genes was only used to conduct genotyping analysis. Keeping the pseudogenes in this gene set gave the genotyping system increased discriminatory power. Indeed, because of their neutral evolution, pseudogenes benefit from a much higher rate of nucleotide substitution than coding sequences [38]. As a result, in our study, the inclusion of pseudogenes made it possible to distinguish strains that could not be distinguished in the phylogenetic analysis based only on 47 coding sequences. Furthermore, almost 20\% of the selected Mccp genes were 
pseudogenes, implying that the analysis of new strains in future genotyping studies may possibly disclose additional pseudogenisation events. On the other hand, for evolutionary analysis, all pseudogenes were discarded. Precise phylogenetic inference and reliable molecular dating were thus based on 47 coding gene sequences presenting similar rates of evolution. Furthermore, detailed sequence analysis did not reveal any mutation hotspots that may have suggested inter-specific recombination. Therefore, the set of genes was considered appropriate for reliable evolutionary analysis.

This multigene approach was applied to a sample of 25 epidemiologically unrelated strains, representing the known global diversity of this species. A large number of stable molecular markers were then selected from this sample by fixing the threshold of polymorphic positions to $85 \%$, therefore excluding potential hypervariable positions. The strategy used here to identify polymorphic positions, based on the comparison of multiple independent strains representing the global species diversity, made it possible to minimise the phylogenetic discovery bias [17].

In the near future, "Whole genome sequencing" (WGS) is expected to become customary for molecular genotyping, since it provides access to information on the entire genome [39]. However, to enable the use of WGS in routine diagnostic laboratories, the main challenge remains the provision of accessible bioinformatics tools to enable the extraction of relevant information out of the enormous amount of data generated, for a fast and reliable analysis $[40,41]$. The use of WGS data from diverse strains would also facilitate new approaches to elucidate the evolution of monomorphic species. However, for evolutionary analyses, the problem is further complicated by the need for reliable molecular clock rates [17].

\section{A new discriminatory system for CCPP epidemiological investigations}

The genotyping system developed here improves robustness and resolution for CCPP epidemiological analysis compared to classical typing methods. The MLST method could not be applied due to insufficient discriminatory power. As an illustration, an MLST scheme based on seven partial housekeeping gene sequences used for the study of the evolution of the M. mycoides cluster revealed only six polymorphic positions between 14. Mccp strains [42]. Seven of these strains were included in our study in which they were distinguished by 135 polymorphisms. As an alternative to MLST, an MLSA system had been developed [4], which remained limited in terms of variability. Our new system, which includes most of the strains previously analysed by MLSA (Table 1), is based on a higher number of polymorphic positions (239 polymorphisms identified in 77
$912 \mathrm{bp}$, versus 53 polymorphisms found in $6747 \mathrm{bp}$ for the MLSA system), resulting in increased discriminatory power (Simpson's index of diversity of 0.997 (0.9881.000) versus 0.964 (0.937-0.991) for the MLSA system).

The six genotyping groups identified in this study (groups A to F; Figure 1) are basically in accordance with MLSA clustering (groups 1 to 5; Table 1) except that diversity analyses now provide evidence for an additional group represented by a single Chinese strain. This strain (M1601) was previously included in MLSA group 3, together with two other strains from the Asian continent, although it branched separately in the MLSA tree, rendering this group polyphyletic. This anomaly was previously accepted because strain M1601 was the only one in the group that did not present a large deletion of around $1000 \mathrm{bp}$, which may have biased distance analysis. However, the present analysis, which is more robust and discriminatory, called for a re-classification of this genotype in a new group (D), the distribution and variability of which remain to be characterised. Most importantly, the intra-group resolution was dramatically increased in the present system, allowing all but two of the strains analysed to be differentiated, while MLSA only afforded further distribution of strains into subgroups, resulting in a biased presentation of the evolutionary distance between strains (Table 1).

A highly discriminatory genotyping tool is now available for the molecular epidemiology of CCPP, allowing precise epidemiological investigations. Recent works have reported the spread of CCPP to new territories, such as the Indian Ocean [9] or the Thrace region of Turkey [10], which constituted a serious threat to Europe. Also, Mccp has been isolated from an increasing variety of wild ungulate species [43-45]. This striking discovery has changed our view of the strict host specificity of Mccp and poses new challenges for CCPP surveillance and control. Tracing new outbreaks requires accurate knowledge of the genetic population structure and the geographic distribution of strains, while the main limitation to such a study remains the paucity of Mccp isolations due to the fastidiousness of this bacterium. However, the use of high throughput sequencing, applied directly on Mccp-rich pleural fluid samples, could circumvent this problem and ease the establishment of a representative Mccp genome database.

\section{The monomorphic bacterium Mccp: a recently emerged pathogen undergoing rapid evolution}

A large-scale genomic approach made it possible to infer the first robust and discriminatory phylogeny of Mccp, to estimate its evolutionary rate, and to date its emergence. A previous study on the molecular evolution of Mccp, based on polymorphisms in the two 16S rRNA gene copies [11], only provided evidence for two major 
lineages for the species. Our study confirmed the presence of these two lineages but, most importantly, it enabled a dramatic increase in the resolution and robustness of the phylogenetic analysis, resulting in the identification of six well-supported clusters. In addition, this multigene approach made it possible to overcome the bias caused by single gene specificities, providing a more reliable inference of the phylogeny of Mccp [46].

The mutation rate of Mccp was estimated by BEAST at $1.3 \times 10^{-6}$ substitutions per site per year, based exclusively on coding gene sequences. This estimate is in agreement with previous reports for other mycoplasma species $[20,47]$, and exceeds the mutation rate estimated for $\mathrm{Mmm}$ at $5.0 \times 10^{-7}$ based on practically the same gene set. Mycoplasmas are among the fastest evolving bacteria, which may be explained by the loss of DNA repair genes during reductive genome evolution [48]. These high mutation rates may facilitate the accumulation of nonsense and frame-shift mutations resulting in gene decay, as evidenced in the analysis of the complete genome of strain 9231-Abomsa, which revealed the presence of 248 pseudogenes representing $25 \%$ of the genome [23]. Increased fixation of detrimental mutations by Mccp may be associated with the severe population bottleneck which characterised the emergence of this species. Indeed, evolutionary and dating analysis made it possible to recreate a plausible scenario for the emergence of the species: i.e., the specialisation of Mcc, a goat pathogen presenting a wider tissue tropism, in a restricted ecological niche (the lung) resulted in the emergence of Mccp. This emergence is indeed a recent event, estimated by BEAST analysis at about 270 years ago (between 120 and 736), in agreement with the estimate of Fischer et al. [42], dating the origin of the species between 56 and 490 years ago. Mccp, like other young pathogens, has evolved too recently to allow the accumulation of many mutations $[49,50]$, thus explaining its low genetic diversity despite a high mutation rate.

\section{Combining large-scale genomic data with spatial and temporal data provides an unprecedented resolution to elaborate plausible scenarios regarding CCPP evolution and epidemiology}

BEAST analysis showed that the ancestor of all Mccp strains emerged about 270 years ago, around 1740 . The first unequivocal accounts of CCPP were reported more than a century after this predicted emergence in Algeria [51] and then in South Africa, involving goats imported from Turkey [52]. It is therefore difficult to establish where this emergence took place simply by examining the literature, particularly since, at that time, СCPP was confused with diseases caused by other mycoplasmas of the M. mycoides cluster which frequently infect goats and induce similar clinical signs and lesions. Historical data must therefore be analysed very carefully to ensure that Mccp was indeed the etiologic agent.

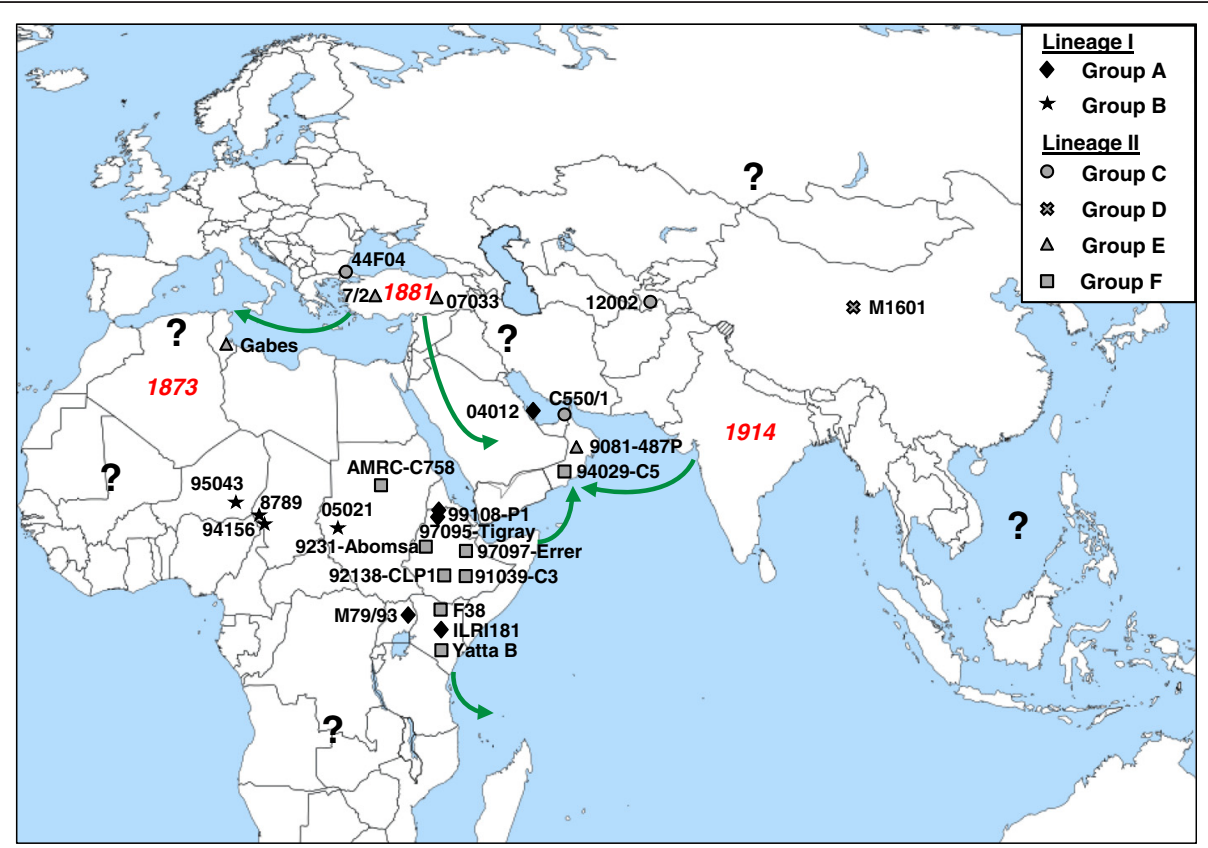

Figure 3 Integration of genomic, geographic, historical and animal movement data to investigate the epidemiology of CCPP. The original location of each Mccp strain is indicated by a symbol, according to the corresponding genotyping group. The green arrows show major CCPP diffusion routes. The dates of the first clinical descriptions of the disease are indicated in red italics. Question marks are placed in areas from where no recent data is available. 
Molecular dating indicated that the MRCA of group C (including strains from Tajikistan, the Arabian Peninsula and Turkey) and group D (represented by one strain from China) (Figure 3) emerged about 200 years ago, around 1810 , indicating that CCPP was present for a long time in Asia. The first historical description of CCPP on this continent may well be that made by Walker in India in 1914 [53]. Molecular dating and historical data therefore suggest that the Asian continent may be the cradle of CCPP. In the Arabian Peninsula, most of the genotyping groups were observed, representing both evolutionary lineages (Figure 3). This finding is not surprising, since this region is known to extensively import animals from various origins, particularly for Muslim feasts [16]. On the other hand, Turkey has been known to export animals to many different areas for a long time [52]. In the present study, two different groups were identified in this country (Figure 3). While the occurrence of group E in North Africa and Turkey is in agreement with animal movements reflecting well known Mediterranean trading routes established during the Ottoman Empire, the identification of group $\mathrm{C}$ in Turkey reveals the great complexity of animal movements in this region. The scarcity of strains from the West, but also from Central and East Asia hampers the precise determination of the emergence, diversity and distribution of Mccp in Asia.

In East Africa, two distinct groups (A and F) were found, each belonging to a different lineage (Figure 3), with MRCAs estimated around 35 and 90 years ago, respectively. Intra-group variability was extremely low for group A strains, while Group F strains showed higher genetic diversity, in agreement with longer evolution times allowing further clonal expansion. The presence of two extremely divergent groups in East Africa suggests that Mccp emerged in this region on at least two different occasions. Strains from both groups then spread thanks to trade and other animal movements between neighbouring countries. However, the origin and dynamics of co-evolution of these two distinctive groups in East Africa remain to be elucidated.

In Central Africa, a strict correlation between genotype (group B) and geographic origin was identified. In addition, the four strains belonging to this group showed very little genetic diversity and the date of their MRCA was estimated at around 40 years ago, suggesting that Mccp must have emerged very recently in Central Africa, as stated by Lefevre et al. when the first strain was isolated in Chad in 1987 [54]. In fact, CCPP was not previously suspected by veterinarians established in this region during the colonial times, although they were familiar with the typical clinical picture of this disease, which was described in scientific journals and reference works [55]. If we consider the distribution of other contagious diseases of goats, such as the "peste des petits ruminants", the presence of CCPP may also be suspected in West Africa. An active search involving improved epidemiological surveillance networks will be required to determine the western limits of CCPP distribution in Africa.

In conclusion, this study illustrates how using a large scale genomic approach can provide an unprecedented resolution to analyse the dynamics and evolution of Mccp. Combining high-throughput genomic data with spatial and temporal data enabled a comprehensive view of the epidemiology of CCPP, which will facilitate the development of improved disease surveillance and control measures. Further efforts are now needed to better define the genetic diversity and distribution of Mccp, particularly in North and Central Africa and in Asia, from where very few strains have been isolated to date.

\section{Additional files}

Additional file 1: List of the 57 genes analysed according to the genome of Mccp strain 9231-Abomsa. The 57 genes selected for analysis are described according to the genome of strain 9231-Abomsa.

Additional file 2: Distribution of selected genes in the genome of Mccp strain 9231-Abomsa. Illustration of the 57 selected genes evenly distributed along the chromosome of strain 9231-Abomsa.

Additional file 3: Mccp strain 9231-Abomsa gene set reference sequence. This reference sequence was used to automatically retrieve the entire gene set from NGS raw data. It consists in the 57 annotated genes from Mccp strain 9231-Abomsa separated by flanking regions (107.050 bp).

Additional file 4: Alignment of 57 concatenated genes of 25 Mccp strains. Input file for genotyping analysis.

Additional file 5: Alignment of 47 concatenated coding sequences of 25 Mccp strains and an Mcc outgroup. Input file for phylogenetic analysis.

Additional file 6: List of polymorphic positions. Description of the polymorphic positions found in the genotyping dataset.

Additional file 7: Phylogeny of Mycoplasma capricolum subsp. capripneumoniae inferred with the maximum likelihood method. The maximum likelihood tree was reconstructed using PhyML based on the alignment of the concatenated sequences of 47 coding sequences from 25 Mccp strains. Bootstrap values $>90 \%$ are shown. The branch corresponding to the outgroup (California Kid) was shortened, as indicated by parallel bars. The scale bar shows the number of substitutions per site.

\section{Competing interests}

The authors declare that they have no competing interests.

\section{Authors' contributions}

VD conceived and designed the study, carried out the experiments and the analysis, drafted the manuscript. LMS coordinated the study and participated in drafting the manuscript. FTH conceived the study and corrected the manuscript. AV developed the bioinformatics in-house software. All authors read and approved the final manuscript.

\section{Acknowledgements}

The authors wish to thank Caroline COSTE for supervision of bioinformatics development, Dr Amirbekov and Dr Ostrowski for providing the sample from which strain 12002 was isolated. This work was supported by the high performance cluster of the UMR AGAP CIRAD.

Received: 10 February 2015 Accepted: 18 May 2015

Published online: 06 July 2015 


\section{References}

1. OIE, Contagious caprine pleuropneumonia, Chapter 2.7.6. in: OIE Terrestrial Manual [on line] (2014). http://www.oie.int/fileadmin/Home/eng/ Health_standards/tahm/2.07.06_CCPP.pdf

2. Manso-Silvan L, Vilei EM, Sachse K, Djordjevic SP, Thiaucourt F, Frey J (2009) Mycoplasma leachii sp. nov. as a new species designation for Mycoplasma sp. bovine group 7 of Leach, and reclassification of Mycoplasma mycoides subsp. mycoides LC as a serovar of Mycoplasma mycoides subsp. capri. Int J Syst Evol Microbiol 59:1353-1358

3. MacOwan KJ, Minette JE (1976) A mycoplasma from acute contagious caprine pleuropneumonia in Kenya. Trop Anim Health Prod 8:91-95

4. Manso-Silvan L, Dupuy V, Chu Y, Thiaucourt F (2011) Multi-locus sequence analysis of mycoplasma capricolum subsp. capripneumoniae for the molecular epidemiology of contagious caprine pleuropneumonia. Vet Res 42:86

5. Nicholas R, Churchward C (2012) Contagious caprine pleuropneumonia: new aspects of an old disease. Transbound Emerg Dis 59:189-196

6. Amirbekov M, Murvatulloev S, Ferrari G (2010) Contagious caprine pleuropneumonia detected for the first time in Tajikistan. EMPRES Transboundary Anim Dis Bull 35:20-22

7. Awan MA, Abbas F, Yasinzai M, Nicholas RA, Babar S, Ayling RD, Attique MA, Ahmed Z, Wadood A, Khan FA (2010) First report on the molecular prevalence of Mycoplasma capricolum subspecies capripneumoniae (Mccp) in goats the cause of contagious caprine pleuropneumonia (CCPP) in Balochistan province of Pakistan. Mol Biol Rep 37:3401-3406

8. Li Y, Zhang JH, Hu SP, Wang L, Xin JQ (2007) Reclassification of the four China isolated strains of the pathogen for contagious caprine pleuropneumonia. Wei Sheng Wu Xue Bao 47:769-773 (in Chinese)

9. Srivastava AK, Meenowa D, Barden G, Salguero FJ, Churchward C, Nicholas RA (2010) Contagious caprine pleuropneumonia in Mauritius. Vet Rec 167:304-305

10. Ozdemir U, Ozdemir E, March JB, Churchward C, Nicholas RA (2005) Contagious caprine pleuropneumonia in the Thrace region of Turkey. Vet Rec 156:286-287

11. Pettersson B, Bolske $G$, Thiaucourt F, Uhlen M, Johansson KE (1998) Molecular evolution of Mycoplasma capricolum subsp. capripneumoniae strains, based on polymorphisms in the 165 rRNA genes. J Bacteriol 180:2350-2358

12. Woese CR (1987) Bacterial evolution. Microbiol Rev 51:221-271

13. Pettersson B, Leitner T, Ronaghi M, Bolske G, Uhlen M, Johansson KE (1996) Phylogeny of the Mycoplasma mycoides cluster as determined by sequence analysis of the $16 \mathrm{~S}$ rRNA genes from the two rRNA operons. J Bacteriol 178:4131-4142

14. Manso-Silvan L, Perrier X, Thiaucourt F (2007) Phylogeny of the Mycoplasma mycoides cluster based on analysis of five conserved protein-coding sequences and possible implications for the taxonomy of the group. Int J Syst Evol Microbiol 57:2247-2258

15. Maiden MC, Bygraves JA, Feil E, Morelli G, Russell JE, Urwin R, Zhang Q Zhou J, Zurth K, Caugant DA, Feavers IM, Achtman M, Spratt BG (1998) Multilocus sequence typing: a portable approach to the identification of clones within populations of pathogenic microorganisms. Proc Natl Acad Sci U S A 95:3140-3145

16. Lorenzon S, Wesonga H, Ygesu L, Tekleghiorgis T, Maikano Y, Angaya M, Hendrikx P, Thiaucourt P (2002) Evolution of M.capricolum subsp. capripneumoniae strains and molecular epidemiology of contagious caprine pleuropneumonia. Vet Microbiol 85:111-123

17. Achtman M (2008) Evolution, population structure, and phylogeography of genetically monomorphic bacterial pathogens. Annu Rev Microbiol 62:53-70

18. Roumagnac P, Weill FX, Dolecek C, Baker S, Brisse S, Chinh NT, Le TA, Acosta CJ, Farrar J, Dougan G, Achtman M (2006) Evolutionary history of Salmonella typhi. Science 314:1301-1304

19. Morelli G, Song Y, Mazzoni CJ, Eppinger M, Roumagnac P, Wagner DM, Feldkamp M, Kusecek B, Vogler AJ, Li Y, Cui Y, Thomson NR, Jombart T, Leblois R, Lichtner P, Rahalison L, Petersen JM, Balloux F, Keim P, Wirth T, Ravel J, Yang R, Carniel E, Achtman M (2010) Yersinia pestis genome sequencing identifies patterns of global phylogenetic diversity. Nat Genet 42:1140-1143

20. Dupuy V, Manso-Silvan L, Barbe V, Thebault P, Dordet-Frisoni E, Citti C, Poumarat F, Blanchard A, Breton M, Sirand-Pugnet P, Thiaucourt F (2012) Evolutionary history of contagious bovine pleuropneumonia using next generation sequencing of Mycoplasma mycoides Subsp. mycoides "Small Colony". PLoS One 7:e46821
21. Roetzer A, Diel R, Kohl TA, Ruckert C, Nubel U, Blom J, Wirth T, Jaenicke $S$, Schuback S, Rusch-Gerdes S, Supply P, Kalinowski J, Niemann S (2013) Whole genome sequencing versus traditional genotyping for investigation of a Mycobacterium tuberculosis outbreak: a longitudinal molecular epidemiological study. PLoS Med 10:e1001387

22. Chu Y, Gao P, Zhao P, He Y, Liao N, Jackman S, Zhao Y, Birol I, Duan X, Lu Z (2011) Genome sequence of Mycoplasma capricolum subsp. capripneumoniae strain M1601. J Bacteriol 193:6098-6099

23. Dupuy V, Thiaucourt F (2014) Complete Genome Sequence of Mycoplasma capricolum subsp. capripneumoniae Strain 9231-Abomsa. Genome Announc 2:e01067-14

24. Falquet L, Liljander A, Schieck E, Gluecks I, Frey J, Jores J (2014) Complete Genome Sequences of Virulent Mycoplasma capricolum subsp. capripneumoniae Strains F38 and ILRI181. Genome Announc 2:e01041-14

25. Lorenzon S, Manso-Silvan L, Thiaucourt F (2008) Specific real-time PCR assays for the detection and quantification of Mycoplasma mycoides subsp. mycoides SC and Mycoplasma capricolum subsp. capripneumoniae. Molecul Cell Probes 22:324-328

26. Sambrook J, Fritsch EF, Maniatis T (1989) Molecular Cloning: A Laboratory Manual. Cold Spring Harbor, New York

27. Galaxy - Data intensive biology for everyone [on line] http://galaxyproject.org/

28. Librado P, Rozas J (2009) DnaSP v5: a software for comprehensive analysis of DNA polymorphism data. Bioinformatics 25:1451-1452

29. Bandelt HJ, Forster P, Rohl A (1999) Median-joining networks for inferring intraspecific phylogenies. Mol Biol Evol 16:37-48

30. Hunter PR, Gaston MA (1988) Numerical index of the discriminatory ability of typing systems: an application of Simpson's index of diversity. J Clin Microbiol 26:2465-2466

31. Grundmann H, Hori S, Tanner G (2001) Determining confidence intervals when measuring genetic diversity and the discriminatory abilities of typing methods for microorganisms. J Clin Microbiol 39:4190-4192

32. Posada D, Crandall KA (1998) MODELTEST: testing the model of DNA substitution. Bioinformatics 14:817-818

33. Guindon S, Dufayard JF, Lefort V, Anisimova M, Hordijk W, Gascuel O (2010) New algorithms and methods to estimate maximum-likelihood phylogenies: assessing the performance of PhyML 3.0. Syst Biol 59:307-321

34. Drummond AJ, Rambaut A (2007) BEAST: Bayesian evolutionary analysis by sampling trees. BMC Evol Biol 7:214

35. Kass RE, Raftery AE (1995) Bayes Factors. J Am Stat Assoc 90:773-795

36. Suchard MA, Weiss RE, Sinsheimer JS (2001) Bayesian selection of continuous-time Markov chain evolutionary models. Mol Biol Evol 18:1001-1013

37. Baker S, Hanage WP, Holt KE (2010) Navigating the future of bacterial molecular epidemiology. Curr Opin Microbiol 13:640-645

38. Li WH, Gojobori T, Nei M (1981) Pseudogenes as a paradigm of neutral evolution. Nature 292:237-239

39. Eyre DW, Golubchik T, Gordon NC, Bowden R, Piazza P, Batty EM, Ip CL Wilson DJ, Didelot X, O'Connor L, Lay R, Buck D, Kearns AM, Shaw A, Paul J, Wilcox MH, Donnelly PJ, Peto TE, Walker AS, Crook DW (2012) A pilot study of rapid benchtop sequencing of Staphylococcus aureus and Clostridium difficile for outbreak detection and surveillance. BMJ Open 2:e001124

40. Carrico JA, Sabat AJ, Friedrich AW, Ramirez M, ESCMID Study Group for Epidemiological Markers (ESGEM) (2013) Bioinformatics in bacterial molecular epidemiology and public health: databases, tools and the next-generation sequencing revolution. Euro Surveill 18:20382

41. Sabat AJ, Budimir A, Nashev D, Sa-Leao R, van Dijl J, Laurent F, Grundmann H, Friedrich AW, ESCMID Study Group for Epidemiological Markers (ESGEM) (2013) Overview of molecular typing methods for outbreak detection and epidemiological surveillance. Euro Surveill 18:20380

42. Fischer A, Shapiro B, Muriuki C, Heller M, Schnee C, Bongcam-Rudloff E, Vilei EM, Frey J, Jores J (2012) The origin of the 'Mycoplasma mycoides cluster' coincides with domestication of ruminants. PLoS One 7:e36150

43. Arif A, Schulz J, Thiaucourt F, Taha A, Hammer S (2007) Contagious caprine pleuropneumonia outbreak in captive wild ungulates at Al Wabra Wildlife Preservation, State of Qatar. J Zoo Wild Med 38:93-96

44. Chaber A, Lignereux L, Qassimi MA, Saegerman C, Manso-Silvan L, Dupuy V, Thiaucourt F (2014) Fatal transmission of contagious caprine pleuropneumonia to an Arabian oryx (Oryx leucoryx). Vet Microbiol 173:156-159

45. Yu Z, Wang $T$, Sun $H$, Xia Z, Zhang $K$, Chu D, Xu Y, Xin Y, Xu W, Cheng $K$, Zheng X, Huang G, Zhao Y, Yang S, Gao Y, Xia X (2013) Contagious caprine pleuropneumonia in endangered Tibetan antelope, China, 2012. Emerg Infect Dis 19:2051-2053 
46. Coenye T, Gevers D, Van de Peer Y, Vandamme P, Swings J (2005) Towards a prokaryotic genomic taxonomy. FEMS Microbiol Rev 29:147-167

47. Delaney NF, Balenger S, Bonneaud C, Marx CJ, Hill GE, Ferguson-Noel N, Tsai P, Rodrigo A, Edwards SV (2012) Ultrafast Evolution and Loss of CRISPRs Following a Host Shift in a Novel Wildlife Pathogen, Mycoplasma gallisepticum. PLoS Genet 8:e100251

48. Carvalho FM, Fonseca MM, Batistuzzo De Medeiros S, Scortecci KC, Blaha CA, Agnez-Lima LF (2005) DNA repair in reduced genome: the Mycoplasma model. Gene 360:111-119

49. Kidgell C, Reichard U, Wain J, Linz B, Torpdahl M, Dougan G, Achtman M (2002) Salmonella typhi, the causative agent of typhoid fever, is approximately 50,000 years old. Infect Genet Evol 2:39-45

50. Sreevatsan S, Pan X, Stockbauer KE, Connell ND, Kreiswirth BN, Whittam TS, Musser JM (1997) Restricted structural gene polymorphism in the Mycobacterium tuberculosis complex indicates evolutionarily recent global dissemination. Proc Natl Acad Sci U S A 94:9869-9874

51. Thomas P. (1873) Rapport médical sur le Bou Frida. In: I'Algérie G.g.c.d. (Ed.), Jourdan A, Alger, pp. 35

52. Hutcheon D (1881) Contagious pleuro-pneumonia in angora goats. Vet J 13:171-180

53. Walker GK (1914) Pleuro-pneumonia of goats in the Kangra district, Punjab, India. J Comp Pathol Ther 27:68-71

54. Lefevre PC, Breard A, Alfarouk I, Buron S (1987) Mycoplasma species F 38 isolated in Chad. Vet Rec 121:575-576

55. Curasson G (1936) Maladies à ultravirus. In: Curasson G (ed) Traité de pathologie exotique vétérinaire et comparée. Vigot frères, Paris, pp 590-606

56. Jones GE, Wood AR (1988) Microbiological and serological studies on caprine pneumonias in Oman. Res Vet Sci 44:125-131

\section{Submit your next manuscript to BioMed Central and take full advantage of:}

- Convenient online submission

- Thorough peer review

- No space constraints or color figure charges

- Immediate publication on acceptance

- Inclusion in PubMed, CAS, Scopus and Google Scholar

- Research which is freely available for redistribution 